\section{Iliac Bone Punch for Psoas Muscle Transplants}

Mr. J. C. Griffrths, of the Royal Manchester Children's Hospital, and Mr. E. DeRvin and Mr. O. B. HeYwood, of the Department of Mechanical Engineering, University of Salford, write: Transfer of the psoas muscle from the lesser to the greater trochanter is now a standard procedure in certain paralytic disorders of the hip. The indications for the operation, originally for paralytic hip dislocation, are now widening to include patients without dislocation but with muscular unbalance where the psoas is overactive or unopposed. ${ }^{1}$

In an early attempt to perform an anterolateral transplant ${ }^{2}$ a deep wedge was cut in the blade of the ilium. This technique is now less popular, and the muscle is more often transferred posteriorly through a hole in the blade of the ilium. ${ }^{3}$ Technical difficulties in the procedure include methods of gaining access to the lesser trochanter from an anterior approach, making a suitable hole in the ilium, and attaching the psoas tendon to the posterior aspect of the greater trochanter.

A bone punch has been devised to solve the problem of producing a hole in the ilium, and the prototype has been used successfully on several patients. The advantages of the device are threefold. Firstly, the dissection required is reduced since iess room is needed. In particular, there is less necessity for wide stripping of tissues for access to the posterior aspect of the ilium, since the instrument will cut cleanly through both hard and soft tissues. This may be expected to reduce the need for wide periosteal stripping, which can produce new bone formation between ilium and femur, followed later by hip ankylosis. Secondly, the time needed for the operation is reduced. Thirdly, a circular hole is cut with clean edges. The psoas muscle passes through easily and does not catch on the jagged edges which remain when the hole is made by more conventional means. It has become clear that these edges are the most usual cause of difficulty and that if they are eliminated a smaller hole is quite adequate.

The punch (see illustration) is operated by turning the hand spindle; this drives the bevel wheel, causing the operating punch to move downwards. Any material between the jaws of the instrument is sheared cleanly and collected in the

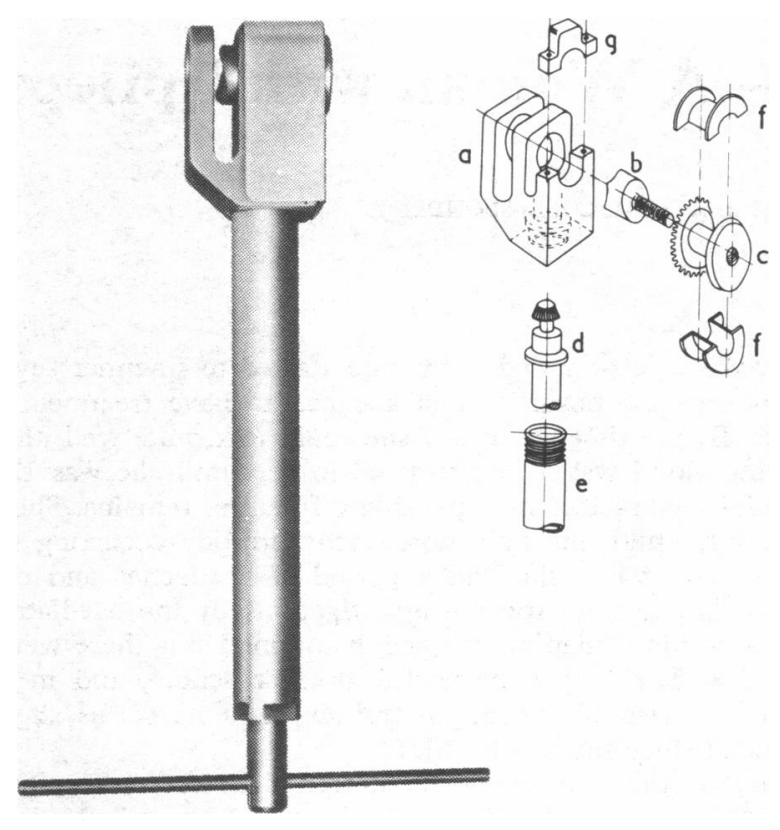

Iliac bone punch: (a) jaw recess; (b) shear punch; (c) bevel wheel drive; (d) pinion driver with driving spindle; (e) handle incorporating spindle bearing; (f) split bearings (P.T.F.E.); (g) bearing cap.

lower jaw aperture. The punch is withdrawn by reversing the rotation of the hand spindle.

All the metal parts of the instrument, including the bevel gears, are made in stainless steel. The bearings are made from a low friction plastic (P.T.F.E.), and the whole instrument can be autoclaved without dismantling or lubrication. The mechanical advantage of the instrument is about 80 to 1 , and the punch shears through the ilium with very little resistance at the operating spindle.

\section{References}

1 Menelaus, M. B., Fournal of Bone and foint Surgery, 1969, 51B, 238.

Mustard, W. T., Fournal of Bone and Foint Surgery, 1952, 34A, 647.

Sharrard, W. J. W., Fournal of Bone and foint Surgery, 1964, 46B, 426.

\section{"Disposable" Tray for Soaking Membranes for Kiil Dialysers}

Dr. F. M. Parsons, consultant in clinical renal physiology, and Mr. B. AUTY, senior artificial kidney technician, Renal Research Unit, General Infirmary at Leeds, write: We first encountered occasional pyrogen-like reactions in haemodialysis in 1956, coming on about 55 to 65 minutes after starting treatment, while using the Kolff-Brigham rotating model. The cause was traced to non-disposable nylon components used in the blood circuit. As nylon absorbs water (about $10 \%$ of its dry weight) we assumed that pyrogen-like substances could also be absorbed to be released later into the blood stream. These reactions ceased when all soiled non- disposable parts were soaked overnight in Haemo-sol and then washed with tap-water in a pipette washer for six hours after each dialysis prior to reautoclaving before assembly for the next treatment.

When we started using Kiil dialysers it seemed that "pyrogen" must be prevented from gaining access to any material capable of absorbing water, particularly the flat-sheet cuprophane membrane, which is preferably wetted before assembly and then chemically sterilized before use. Initially we used autoclaved pyrogen-free $3 \%$ acetic acid for wetting the membrane in a polyethylene tank and for sterilizing the assembled dialyser. Only one pyrogen-like reaction occurred despite bacterial invasion of the dialysis fluid. Later the bacterial count in the dialysis fluid was reduced to insignificant levels by using filtration. The high incidence of infections 
from aerobic spore-bearing organisms, however, made us change the sterilizing fluid from 3\% acetic acid to formalin.

The membranes were then dipped, one at a time, in a polyethylene tank filled with autoclaved pyrogen-free water before laying on the Kiil boards and autoclaved pyrogen-free $1.5 \%$ formaldehyde solution (later $2.5 \%$ ) was used for "sterilization" of the assembled dialyser. Occasional pyrogenlike reactions developed which were abolished when the polyethylene tank was filled with hypochlorite solution between use. Presumably the hypochlorite solution was acting in much the same microbiological manner as the 3\% acetic acid that was used initially. Thus one of the biggest causes of pyrogen-like reactions could, by deduction, be traced to the polyethylene tank used for wetting membranes. Repeated cleaning and filling of this with hypochlorite solution was a messy, expensive, and, in microbiological terms, an unsatisfactory procedure.

A membrane immersion tray with a disposable polyethylene lining sheet was designed. The base of the tray was made from Formica-faced blockboard 120 by 60 by 1.5 $\mathrm{cm}$. The legs fitted to the undersurface of the blockboard folded flush to make storage easier. The free-standing retaining walls of the tray, to provide vertical support to the polyethylene sheet at the edges of the pool of contained water, were made from unplasticized polyvinyl chloride or acronitrile-butadiene-styrene sheet $6 \mathrm{~mm}$ thick, the two strips forming the longer walls were 120 by $5 \mathrm{~cm}$, the two forming the shorter walls being 60 by $5 \mathrm{~cm}$. Three centimetres from the end of each of the four walls slots $2.5 \mathrm{~cm}$ deep and 6.5 $\mathrm{mm}$ wide (see diagram) were cut so that the walls could be fitted together to form a rectangle approximately 114 by 54 $\mathrm{cm}$.

A sheet of polyethylene (170 by $90 \mathrm{~cm}, 200$ gauge), folded during manufacture in its long length and made presumably by slitting a lay-flat blown-tube, was opened so that the inner "clean" surface formed the upper wetted surface of the liner when placed over the walls resting on the Formica sheet. When partially filled with pyrogen-free water the liner formed a tank adequate for immersion of cuprophane membranes. The polyethylene lining sheet, costing less than $1 \mathrm{p}$, is thrown away after use.

Over 650 dialyses have been undertaken in our hospital unit since the introduction of this disposable polyethylene liner and no pyrogen-like reactions have occurred. The device offers advantages in lower chemical costs, reduction in labour, and smaller storage space.

Despite this advance the microbiological aspects of assembling Kiil dialysers in a hospital unit are unsatisfactory. Even though our technicians wear gloves, the placing of the wet membranes on the open Kiil boards contaminated with, inter
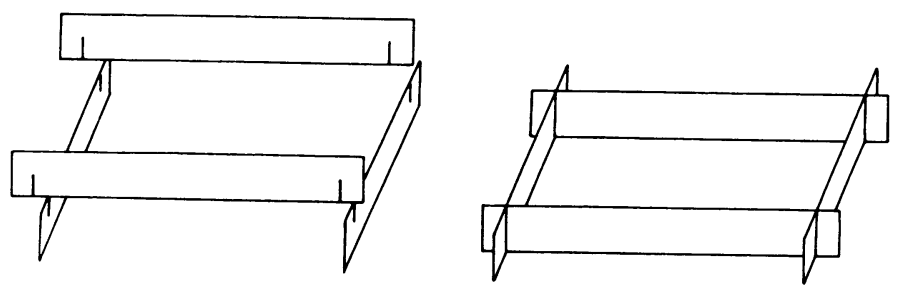

Diagram of retaining walls for the membrane tray.

alia, organisms from previous use and storage ${ }^{2}$ clearly leads to contamination of the outside of the gloves, this can be passed to the liquid in the tray and hence, via the next membranes to be soaked, to subsequent boards in the assembly procedure. Thus the chances of cross-infection occurring between dialysers, if several are being assembled at the same time, are real and serious.

It would seem to us that this form of dialyser, using a wet and possibly pyrogen-free assembly procedure but with contaminated components, must be replaced by a disposable type in which "biologically" clean components are assembled in a dry procedure under conditions of controlled hygiene and physically separated from a potentially infected dialysis area. ${ }^{3}$

\section{References}

1 Parsons, F. M., Shoesmith, J. G., and Flower, J. R., Filtration Society Symposium on Filtration in Medical and Health Engineering, April 1969 p. 22

${ }^{2}$ Jones, D. M., Tobin, B. M., Harlow, G. R., and Ralston, A. J., British Medical fournal, 1970, 3, 135. 3 Flower, J. R., and Parsons, F. M., Proceedings of the European Dialysis
and Transplant Association, 1970, 7, 437 . 